\title{
Creación de página Web para promover aprendizaje colaborativo en alumnos de máster
}

\author{
María González-Béjar ${ }^{\text {ab }}$, Daniel Cuaran-Acosta ${ }^{\mathrm{a}}$, Lorena Andrés-Olmos ${ }^{\mathrm{b}}$, Ignacio \\ Rosa-Pardo $^{\mathrm{b}}$, Raquel del Rincón ${ }^{\mathrm{bc}}$, Elena Zaballos-García ${ }^{\mathrm{a}}$, Julia Pérez-Prieto ${ }^{\mathrm{ab}}$ \\ ${ }^{a}$ Departamento de Química Orgánica, 'Instituto de Ciencia Molecular, Universitat de Valencia, \\ (maria.gonzalez@uv.es), ${ }^{\circ}$ Departamento de Química Inorgánica, Universitat de Valencia.
}

\begin{abstract}
A Web page has been designed by master students in order to engage them in collaborative and cooperative learning, thus improving their learning and comprehension of relevant concepts and topics. To that purpose, topics included in the subject Advanced Techniques in Chemistry which is part of the Master in Sustainable Chemistry of the University of Valencia have been included in the Webpage with the help of WIX software. This approach makes intensive use of the Web as a key technological resource for learning, where the information seen in the subject is summarized and given in an applicative and pertinent context that is easy to understand for all public interested in the topics discussed in there.

This tool allows the development of a key digital competence in the Spanish educational system, articulating the development of the Website with the management of multimedia content. It also allows a sense of initiative and entrepreneurship, since it is of total freedom on the part of the students working in group, the uploaded content, always following the knowledge developed and acquired in the subject.
\end{abstract}

Keywords: collaborative learning, web design, group learning, methodology.

\section{Resumen}

Se ha diseñado una página web para involucrar a los estudiantes de máster en el aprendizaje colaborativo y cooperativo, mejorando así su aprendizaje y comprensión de los temas y conceptos relevantes. Para ello, los temas incluidos en la asignatura Técnicas Avanzadas en Química que forma parte del Máster en Química Sostenible de la Universidad de Valencia se han incluido en la página web con la ayuda del software WIX. Este enfoque hace un uso intensivo de la web como recurso tecnológico clave de aprendizaje, donde se resume la información vista en la asignatura y se le da un contexto aplicativo y pertinente fácil de entender para todo el público interesado en los temas allí tratados.

Esta herramienta permite el desarrollo de una competencia digital clave en el sistema educativo español, articulando el desarrollo de la página web con el manejo de contenido multimedia. Además permite un sentido de iniciativa y emprendimiento, ya que hay libertad por parte de los estudiantes trabajando en grupo, para sellecionar el contenido de la página, siempre siguiendo el 
conocimiento desarrollado y adquirido en la asignatura y el asesoramiento de la profesora.

Palabras clave: aprendizaje colaborativo, diseño página web, aprendizaje en grupo, metodología.

\section{Introducción}

El aprendizaje cooperativo/ colaborativo es un método de enseñanza y aprendizaje en el cual los estudiantes se unen para explorar una pregunta importante o crear un proyecto significativo. (Barkley et al., 2007). Para alcanzar la calidad en la educación es indispensable que los alumnos cooperen para adquirir el aprendizaje necesario. (Torrego y Negro, 2012).

En este contexto, NANOINNOVA es un proyecto de innovación docente que surge de la renovación de metodologías docentes (plan B en la convocatoria de innovación de la Universidad de Valencia de 2017-2018) con el objetivo de desarrollar la capacidad de aprendizaje colaborativo entre los alumnos. (González-Béjar et al., 2017)

Es muy importante utilizar variedad de tecnologías de la comunicación tanto para proporcionar la flexibilidad necesaria para cubrir necesidades individuales y sociales como para lograr entornos de aprendizaje efectivos y una buena interacción entre estudiantes y profesores. (Cabero et al., 2003)

Por ello, esta actividad tiene como objetivo principal utilizar la tarea colaborativa como metodología de enseñanza y aprendizaje. En este contexto, se ha seleccionado como proyecto significativo la creación de una página Web para desarrollar competencias en el marco de un espacio colaborativo entre las alumnas y alumnos de la asignatura de Técnicas Avanzadas en Química del Máster Interuniversitario en Química Sostenible de la Universidad de Valencia, utilizando como material didáctico parte de los materiales docentes de la asignatura y artículos científicos relacionados con dicho contenido. Específicamente, la actividad está dirigida a los alumnos matriculados en la asignatura involucrada en dicho máster en la Universidad de Valencia y se hace referencia tanto a técnicas de separación y purificación como de determinación estructural y caracterización de compuestos orgánicos y nanomateriales.

Además, en la actualidad es cada vez más común utilizar los entornos virtuales de aprendizaje (EVA, por sus siglas en inglés) (Quesada, 2013; Tapscott, 1998), ya que están al alcance de la mayoría y es una forma muy rápida de recopilar gran cantidad de información. Con este tipo de tarea se estimula la creatividad del alumno, debido a que este deja de ser un mero espectador de la clase y pasa a tener un papel más colaborativo, aumentando así la necesidad de intercambiar ideas y negociar opiniones con sus compañeros y/o profesor, entre otros (UNESCO, 2004).

Teniendo en cuenta todo lo mencionado hasta ahora, se han planteado los objetivos descritos a continuación con la finalidad de diseñar una página web para involucrar a los estudiantes de máster en el aprendizaje colaborativo y cooperativo. Esta herramienta permite además el

(cc) EY-NC-ND 2018, Universitat Politècnica de València

Congreso IN-RED (2018) 
desarrollo de una competencia digital clave en el sistema educativo español y favorece la adquisición de competencias del alumnado mediante el trabajo en equipo.

\section{Objetivos}

- Adquirir y afianzar conceptos importantes y competencias en la asignatura.

- Relacionar conocimientos teóricos con aplicaciones reales.

- Simplificar la información a un lenguaje de fácil comprensión.

- Desarrollar competencias tecnológicas.

- Crear una página web con los contenidos más atractivos e innovadores de la asignatura para motivar al estudiantado y reforzar el aprendizaje de competencias.

- Desarrollar la capacidad de trabajo en equipo fomentando:

- La comunicación alumno-profesor y alumno-alumno.

- La responsabilidad del alumno.

- La toma de decisiones.

- La interdependencia positiva.

- La asunción de roles.

\section{Desarrollo de la innovación}

Como parte del desarrollo de la asignatura "Técnicas Avanzadas en Química (TAQ)" del Máster Interuniversitario en Química Sostenible de la Universidad de Valencia, se propuso el desarrollo de una página web donde se expusiera de manera sencilla y clara los contenidos adquiridos. La tarea fue asignada como una única tarea para el grupo de 5 estudiantes, impulsando con ello el trabajo conjunto y la innovación en la asignatura.

El profesor ha facilitado la creación de un clima de aprendizaje y colaboración al despertar el interés de los alumnos alineando los objetivos con los contenidos del temario (Erkens et al., 2006) y ha proporcionado los recursos necesarios para abordar el reto de la creación de contenidos en la página web. Asimismo, se han planificado sesiones de seguimiento para dialogar, razonar y asesorar al estudiantado en la elaboración de la página.

En primer lugar, el estudiantado ha asimilado el contenido de la asignatura mediante asistencia a clases teóricas y prácticas de laboratorio. Cabe destacar que tanto en las clases teóricas como prácticas se ha dado con antelación acceso a recursos docentes preparados por la profesora en el Aula Virtual. De esta forma se ha fomentado un aprendizaje previo basado en el interés de cada participante motivado por la futura creación de la página web.

En este punto, de forma grupal, cada participante ha dialogado y razonado la información que considera adecuada para incluir en la web basándose en los conocimientos previos adquiridos. Se fomenta la comunicación (saber escuchar, comunicar ideas), es decir, el diálogo. Es un método constructivo de aprendizaje dado que el alumnado está implicado. A diferencia de la clase magistral, los alumnos colaboran (Barkley et al. 2007), participan y no son meros receptores de conocimiento. Este proceso permite que el alumno tome conciencia de la interdependencia y repercusión del trabajo de cada componente del grupo. Por otra parte, se fomenta la toma de decisiones ya que los participantes deciden que incluyen y por qué y razonan con la profesora los motivos de la selección de contenidos (Bruffee, 1993). 
La página web fue creada mediante el uso de la plataforma de desarrollo Wix (www.wix.com). Pese a que los conceptos se encuentran al alcance de cualquier buscador, la página web diseñada presenta grandes diferencias: mediante el uso de contenido multimedia permite un aprendizaje más rápido y sencillo, y el uso de artículos científicos como herramienta complementaria a los conceptos permite encontrar una aplicación real de los mismos. El tenerlo todo recopilado en una sola página web hace la búsqueda más sencilla y llamativa para el alumnado y los posibles visitantes de la Wix.

Si bien la obtención de una herramienta útil de trabajo para los alumnos de esta asignatura (página web) es un logro a destacar en el presente proyecto, el mayor logro reside en las competencias que han ido desarrollando en los alumnos a lo largo del proceso en cuanto a la sus capacidades de aprendizaje y también en cuanto a la adquisición de conocimientos propios de la asignatura; altamente relacionado con el proceso de aprendizaje. Merece ser destacado, que el proceso seguido ha permitido, por ejemplo, estimular al alumno y desarrollar un mayor grado de responsabilidad con su trabajo, haciendo que tome conciencia del papel de interdependencia positiva que desarrolla en el equipo.

Tal y como indican Bazo y Luengo, no es suficiente para adquirir una competencia que un alumno sea capaz, cuando se le demanda, de realizar una operación para la que ha sido entrenado o de repetir el enunciado que ha memorizado. "Debe ser capaz, de entre los recursos que se poseen (o que es capaz de buscar), de escoger aquellos que mejor conviene a la situación, para lo cual tendrá que movilizar todos los saberes que posea, tanto explícitos como implícitos, y tendrá que hacerlo mediante los procesos cognitivos adecuados. Una competencia no es la simple adición de conocimientos, sino la capacidad de ponerlos en interacción en función del uso que se le pueda dar en el tratamiento de las situaciones". (Luengo y Bazo, 2009)

Evaluación: Los estudiantes ha de hacer una presentación final una vez concluida la creación de la página. A partir de esta presentación la profesora evalúa el trabajo realizado por los alumnos y la cooperación e implicación que han tenido todos ellos para realizar esta página Web de manera conjunta, eliminando con este método de trabajo el reparto de tareas y la falta de conexión de las partes del trabajo cuando se realiza de manera individual. Además de la evaluación de la profesora, cada alumno deberá evaluar tanto la implicación de cada uno de sus compañeros como la suya propia. De esta manera, al hacer una autocrítica, cada alumno puede reconocer y consensuar su grado de implicación en la asignatura.

\section{Resultados}

Para el desarrollo de la página ha sido necesario un trabajo en equipo, donde cada uno de los integrantes ha aportado sus conocimientos de tecnología y diseño para crear la plataforma base donde se comienza a incluir la información. La página web diseñada se encuentra disponible para libre acceso en el siguiente link: $\leq$ https://taquv17.wixsite.com/nanoparticles $>$, el cual a su vez se publicará en la página oficial 
del Máster en Química Sostenible de la Universidad de Valencia, con lo que se mejora el público objetivo de la web.

La web será una herramienta en constante desarrollo y actualización por parte de los estudiantes de TAQ, quienes dispondrán de acceso como administradores y podrán modificar, complementar y/o actualizar la web para evitar su obsolescencia.

En la Figura 1 se observa la portada de la web. Mediante la inclusión de algunos botones y un video descriptivo del tema general, se plantea de manera intuitiva el contenido que podrá allí encontrarse.

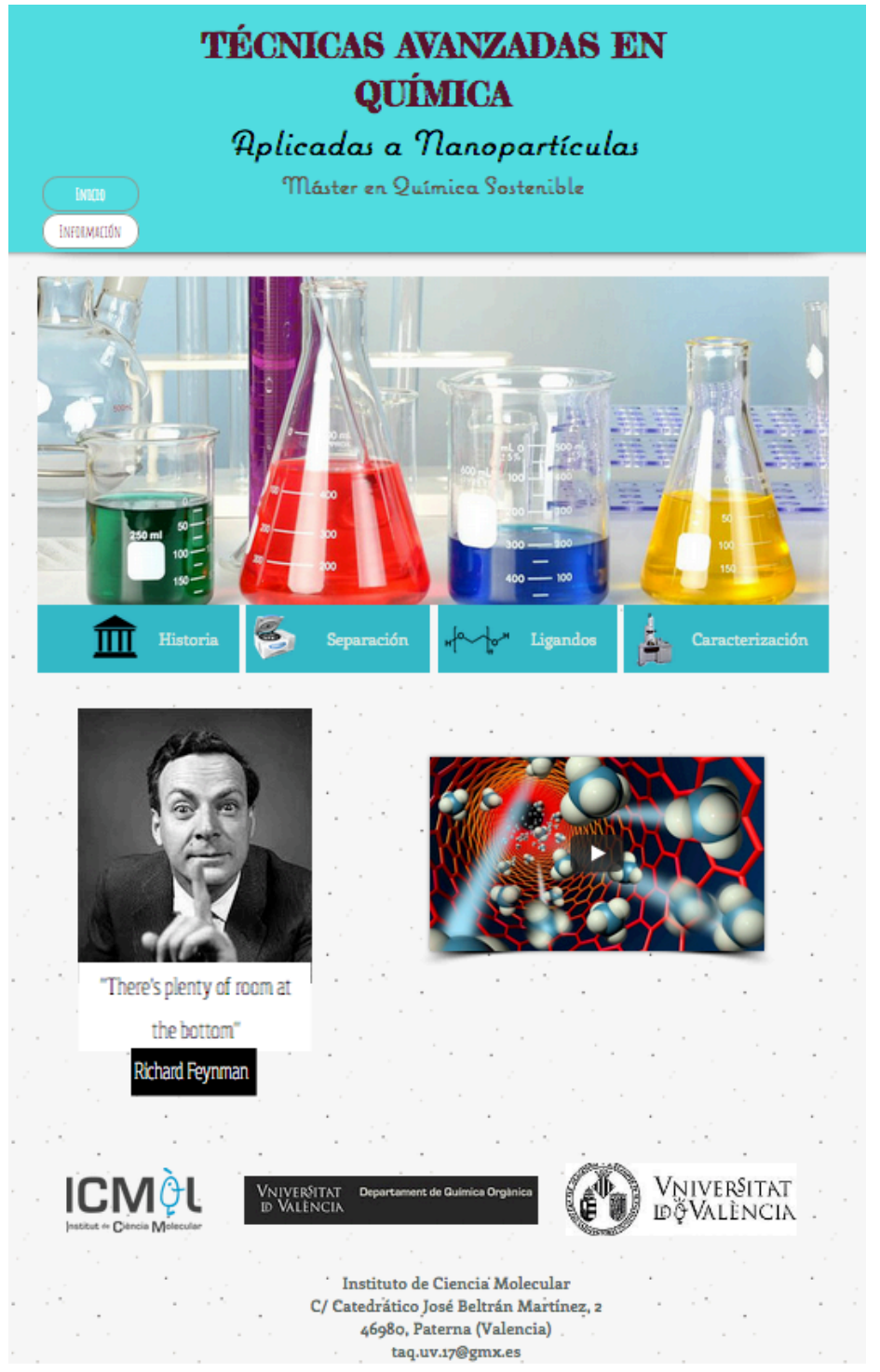

Fig. 1 Portada de la página Web diseñada. 
Para el contenido temático enlazado en cada apartado, se requirió de conocimiento teórico adquirido en la asignatura, así como elementos multimedia de YouTube u otras páginas web y la inclusión de elementos PDF de libre acceso.

Al final, como se observa en las Figuras 2 y 3, para cada temática se incluyen 3 factores clave: multimedia, conceptos e información adicional.

Multimedia: Videos, esquemas, tablas o imágenes.

Conceptos: Información teórica sencilla y corta para dar un panorama general del tema a tratar.

Información adicional: Información asociada mediante hipervínculos, que puede ser de interés para ampliar el conocimiento del tema, tales como handbooks, tesis doctorales, libros y artículos científicos de libre acceso, entre otros.

\section{ESPECTROSCOPIA FOTOELECTRÓNICA DE RAYOS X (XPS)}
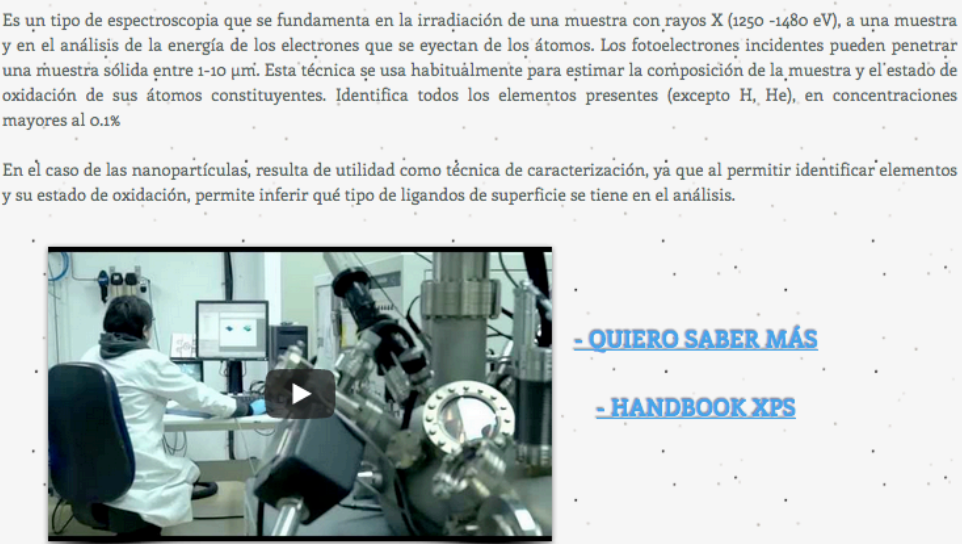

\section{MICROSCOPIA ELECTRÓNICA DE TRANSMISIÓN (TEM)}

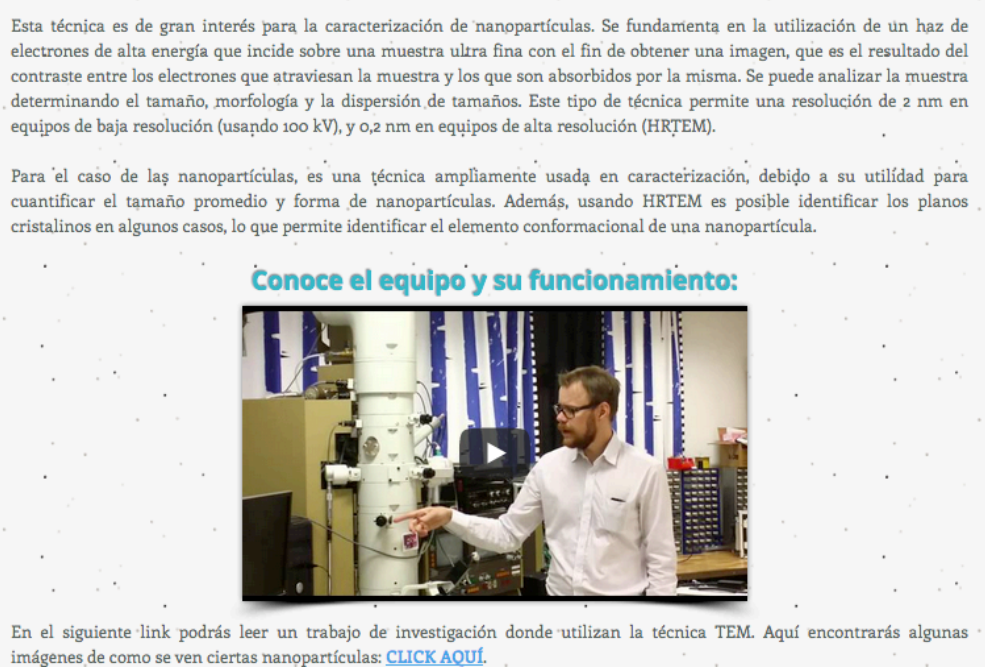

imágenes de como se ven ciertas nanopartículas: CLICK AOUf

Fig. 2. Ejemplos de un tema incluido en la web, donde se puede observar una breve explicación de las técnicas, así como un video ilustrativo del proceso. 


\section{TÉCNICAS AVANZADAS EN} QUÍMICA

\section{LIGANDOS DE NANOPARTÍCULAS METÁLICAS}

Las nanopartículas metálicas presentan una elevadísima área superficial, por lo que pueden ser fácilmente funcionalizadas y bioçonjugadas, modificando sụs propiedades superficialęs. Por ejemplo, una nanopartícula de oro (AuNP), de anm de diámetro de núcleo podría, en principio, ser conjugada a $\sim 100$ moléculas en los ligandos disponibles $(n=\sim 108)$. De hecho, recientemente Gibson y col. anclaron 70 moléculas del agente quimioterapéutico paclitaxel en una AuNP de anm de diámetro.

La funcionalización de las AuNPs se realiza generalmente mediante enlaces «tiol», si bien también muestran afinidad por los grupos amino, fosfito y bisulfito. Numerosas moléculas han sido ligadas a la superficie de las AuNPs con distintos propósitos, ampliando su rango de aplicación. Se pueden utilizar procedimientos de modificación post-sintética para funcionalizar la superficie de la partícula con porciones biocompatibles tales como oligo(etilenglicol) (OEG) y poli(etilenghicol) (PEG).

Ejemplos de estrategias de funcionalización de las AuNPs de aphicación en terapia del cáncer son la conjugación con PEGtiolado para conseguir una vectorización pasiva hacia células tumorales (aumenta el tiempo de vida media en el torrente sanguíneo y muestra una mejora de la permeabilidad y del efecto de retención en el tumor); y la utilización de anticuerpos específicos de biomarcadores moleculares sobrẹexpresados en là superfiçe de células cancerígenas,o la utilización de. péptidos con propiedades de reconocimiento específico de componentes intracelulares. Estos pueden ser conjugados directamente a las AuNPs o mediante puentes de PEG tiolado.

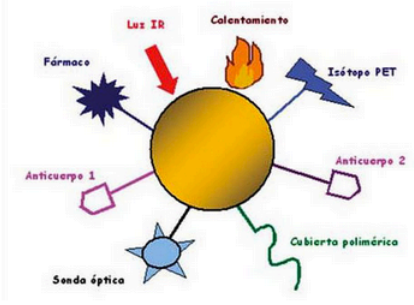

Imagen 1. NP esférica multifuncionalizada con posibles ligandos: fármacos, anticuerpos de vectorización, polímero, sondas de diagnóstico por imagen, etc.

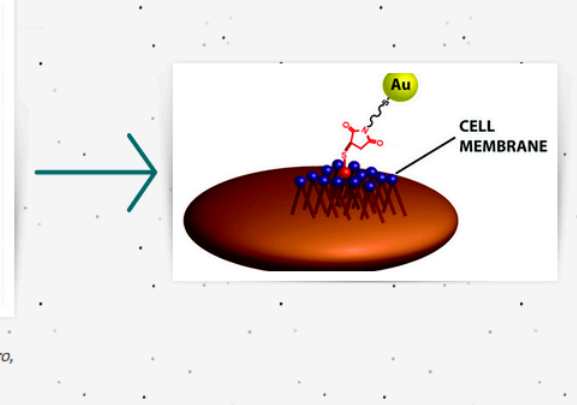

Para mayor comprensión, en el siguiente link podrás encontrar un trabajo doctoral realizado en la Universidad de la Rioja, donde sintetizan nañopartículas de oro y plata con diferentes ligandos orgánicos, dándole una aplicabilidad real a los conceptos aquí indicados: VER TESIS

Los ligandos se encuentran entre las nanopartículas y el medio en el que se encuentran. Por ello, forman parte de las. interacciones entre las nanopartículas y su entorno. Además muchas aplicaciones se basan o depende del intercambio de ligandos, El intecambio que puede llevarse a cabo mediante ditintas formas:

Fig. 3. Ejemplo de otro tema incluido en la web, donde se puede observar una breve explicación de la técnica, así como enlaces a materiales de interés en internet.

Los resultados del aprendizaje abordan todos los niveles cognitivos de la taxonomía de Bloom son: conocer, comprender, aplicar, analizar, evaluar y crear. (Peña, 2010).

\section{Conclusiones}

El alumnado de máster ha creado trabajando en grupo una página web estimulante y motivadora donde se puede encontrar de forma rápida, clara y sencilla los contenidos más relevantes de la asignatura de Técnicas Avanzadas en Química, en concreto la mayoría de 
técnicas e información necesaria para trabajar con nanomateriales, especialmente nanopartículas.

Al realizar este trabajo de forma conjunta utilizando la creación de la página web como catalizador para promover la enseñanza-aprendizaje, cada participante se ha involucrado, intercambiado ideas expresando opiniones y, en grupo han sido capaces de escoger entre los recursos que se poseen (o que se han buscado) movilizando así todos los saberes que posee, tanto explícitos como implícitos para conseguir una visión global de todo el contenido de la asignatura y es capaz de explicar y encontrar información relativa a las técnicas estudiadas.

Además, se han adquirido competencias digitales clave en el sistema educativo español, articulando el desarrollo de la página web con el manejo de contenido multimedia.

Cabe destacar que todos los estudiantes implicados destacan la mayor facilidad de asimilación de contenidos de la asignatura en forma global y el incremento del interés y la motivación en su proceso de aprendizaje.

\section{Referencias}

BARKLEY, E.F., CROSS, K. P., \& MAJOR, C. H. (2007). Técnicas de aprendizaje colaborativo: manual para el profesorado universitario. Madrid: Editorial Morata.

BRUFFEE, K. A. (1998). Collaborative Learning: Higher Education, Interdependence, and the Authority of Knowledge (2nd edition). Baltimore, Md: Johns Hopkins University Press.

CABERO, J., CASTAÑO, C., CEBREIRO, B., GISBERT, M., MARTINEZ, F., MORALES, J. A., SALINAS, J. (2003). Las nuevas tecnologias en la actividad universitaria. Píxel-Bit. Revista de medios y educación, 20, 81-100.

ERKENS, C., JAKICIC, C., JESSIE, L., KING, D., KRAMER, S. V., TWADELL, E. (2008). The Collaborative Teacher: Working Together as a Professional Learning Community. Bloomington, IN: Solution Tree.

GONZÁLEZ-BÉJAR, M., GALIAN, R. E., PÉREZ-PRIETO, J., ZABALLOS-GARCÍA, FRANCÉS SORIANO, L., GONZÁLEZ CARRERO, S., y ESTEBANEZ, N. (2017-2018). NANOINNOVA: Aprendizaje colaborativo en síntesis y caracterización de nanomateriales, Proyecto de Innovació educativa de la Universitat de València (UV-SFPIE_RMD17-588248), Vicerectorat de Polítiques de Formació i Qualitat Educativa Valencia (ESPAÑA)

LUENGO, F., \& BAZO, P. (2009). La práctica de la comunicación lingüistica Proyecto Atlántida.

PEÑA, J. (2010). El aprendizaje cooperativo y las competencias. Revista d'Innovació Docent Universitària, 2, 1-9. 10.1344/105.000001520

QUESADA, A. (2013). Aprendizaje colaborativo en entornos virtuales: los recursos de la Web 2.0. Revista de Lenguas Modernas, (18), 337.

TAPSCOTT, D. (1998). Growing Up Digital: The Rise of the Net Generation. McGraw Hill, Nueva York.

TORREGO, J. C., NEGRO, A. (2012). Aprendizaje cooperativo en las aulas: fundamentos y recursos para su implantación. Madrid: Alianza.

UNESCO. (2004). Las tecnologías de la información y la comunicación en la formación docente: Guía de Planificación. Traducción TRÍAS y ARDANS.

(cc) EY-NC-ND 2018, Universitat Politècnica de València

Congreso IN-RED (2018) 